\title{
Nanorice: a Hybrid Plasmonic Nanostructure
}

\author{
Hui Wang, ${ }^{1,4}$ Daniel W. Brandl, ${ }^{2,4}$ Fei Le, ${ }^{2,4}$ Peter Nordlander, ${ }^{2,3,4}$ Naomi J. Halas*,1,3,4 \\ ${ }^{1}$ Department of Chemistry, ${ }^{2}$ Department of Physics and Astronomy, ${ }^{3}$ Department of Electrical and \\ Computer Engineering, and ${ }^{4}$ the Laboratory for Nanophotonics, Rice University, Houston, TX, 77005 \\ * To whom correspondence should be addressed. Email: halas@rice.edu
}

\section{Experimental details:}

Ferric chloride $\left(\mathrm{FeCl}_{3} \bullet 6 \mathrm{H}_{2} \mathrm{O}\right)$, (3-aminopropyl)trimethoxysilane (APTMS, 97\%), tetrachloroauric acid $\left(\mathrm{HAuCl}_{4} \cdot 3 \mathrm{H}_{2} \mathrm{O}\right)$, tetrakis hydroxymethyl phosphonium chloride (THPC) were purchased from Sigma-Aldrich (St. Louis, MO). 37\% formaldehyde, potassium dihydrogen phosphate $\left(\mathrm{KH}_{2} \mathrm{PO}_{4}\right)$ and 200-proof ethanol were obtained from Fisher Scientific (Hampton, NH). All the chemicals were used as received without further purification. Ultrapure water (18.2 M $\Omega$ resistivity) was obtained from a Milli-Q water purification system (Millipore, Billerica, MA).

Monodisperse spindle-shaped hematite particles with controllable aspect ratios were fabricated by forced hydrolysis of ferric chloride solutions. ${ }^{1}$ Hematite particles with aspect ratio of 6.3 (340 nm x 54 $\mathrm{nm}$ ) were prepared by aging $100 \mathrm{~mL}$ of aqueous solution containing $2.0 \times 10^{-2} \mathrm{M} \mathrm{FeCl}_{3}$ and $4.0 \times 10^{-4} \mathrm{M}$ $\mathrm{KH}_{2} \mathrm{PO}_{4}$ at $100{ }^{\circ} \mathrm{C}$ for 72 hours. The resulting precipitate was centrifuged and washed several times with water and ethanol. Finally, the precipitate was redispersed in $25 \mathrm{~mL}$ ethanol.

The seed particles used in the present study were fabricated following a similar procedure as the previously reported method for the immobilization of Au nanoparticles on silica surfaces. The surface of the spindle-shaped hematite particles was functionalized with organosilane molecules (APTMS) to generate an amine moiety coated surface. Typically, $600 \mu \mathrm{L}$ of APTMS was introduced into $5 \mathrm{~mL}$ of ethanolic solution of hematite particles under vigorous stir. The surface functionalization of hematite particles were accomplished by stirring the mixture for 12 hours. The resulting particles were centrifuged and redispersed in ethanol several times to get rid of the excess APTMS. THPC-capped Au nanoparticles $\left(\sim 2 \mathrm{~nm}\right.$ in diameter) were prepared following Duff's method, ${ }^{2}$ and then attached to the functionalized hematite particle surfaces through the gold-amine interactions. ${ }^{3}$ These attached $\mathrm{Au}$ colloids acted as the nucleation sites for the reduction of $\mathrm{Au}$ ions from solution onto the hematite surface until continuous and complete Au nanoshells were formed. ${ }^{4}$

Nanorice particles were fabricated via seed-catalyzed reduction of $\mathrm{AuCl}_{4}{ }^{-}$ions by formaldehyde in 
aqueous solutions at room temperature. The growth of complete Au shells typically took 5-10 min. By adjusting the amount ratio between seed particles and $\mathrm{AuCl}_{4}{ }^{-}$ions, the thickness of the Au shells can be precisely controlled. The resulting nanorice particles can be homogenously dispersed in water to form colloidal solutions.

Nanorice films were prepared by immobilizing nanorice particles on polyvinylpyridine (PVP)-coated glass substrates. ${ }^{5,6}$ Briefly, glass slides were cleaned in piranha solution (sulfuric acid : hydrogen peroxide, 7:3) and immersed in a 1\% wt. solution of PVP in ethanol for 24 hours. The slides were rinsed thoroughly with ethanol and dried with $\mathrm{N}_{2}$ gas. The PVP-functionalized slides were immersed in an aqueous solution of nanorice for 1 hour. Upon removal from the nanorice solution, the films were rinsed with ethanol and dried with $\mathrm{N}_{2}$. This resulted in a monolayer of isolated nanorice particles with random orientations.

Scanning electron microscope (SEM) images were obtained on a Phillips FEI XL-30 environmental scanning electron microscope at an acceleration voltage of $30 \mathrm{kV}$. The samples for SEM measurements were prepared by drying a drop of colloidal solutions on silicon wafer surface under ambient air. TEM images were obtained using JEOL JEM-2010 transmission electron microscope. The extinction spectra were obtained using a Cary $5000 \mathrm{UV} /$ Vis/NIR spectrophotometer in the wavelength range of $400 \mathrm{~nm}$ to $2400 \mathrm{~nm}$.

\section{Plasmon hybridization:}

The plasmon hybridization method can be used to describe the plasmon modes of the nanorice particles in a manner analogous to the plasmon modes in the spherical nanoshell system. ${ }^{7}$ The plasmons can be viewed as resulting from hybridization of the aspect-ratio-dependent primitive plasmon modes of a solid metallic prolate spheroid and the primitive plasmon modes of a prolate spheroidal dielectric cavity in a uniform metallic background. For simplicity we assume a uniform metallic electron density of $\mathrm{n}_{0}$ corresponding to a bulk plasmon energy $\omega_{B}=\sqrt{\frac{4 \pi n_{0} e^{2}}{m_{e}}}$.

The scalar potential used in the plasmon hybridization method to describe the deformations of the electron liquid in a prolate spheroidal system takes the form: ${ }^{8}$

$\eta=\sum_{l m}\left[\dot{S}_{l m}(t) P_{l m}(\cosh \alpha)+\dot{C}_{l m}(t) Q_{l m}(\cosh \alpha)\right] P_{l m}(\cos \beta) e^{i m \phi}$

where $\mathrm{S}_{\mathrm{lm}}$ are the amplitudes for the primitive plasmons of the solid particle, and $\mathrm{C}_{\mathrm{lm}}$ are the amplitudes for the primitive plasmons of a cavity. The functions $\mathrm{P}_{1 \mathrm{~m}}$ and $\mathrm{Q}_{\operatorname{lm}}$ are the associated Legendre 
polynomials of the first and second kind. In prolate spheroidal coordinates, the surface of constant $\alpha$ is an elliptical spheroid, and coth $\alpha$ is the aspect ratio of the spheroid that is formed. ${ }^{8}$

Prolate spheroidal coordinates are defined with a unique focal length, and the nanorice particles are modeled by confocal ellipsoidal shells. The outer and inner surface of the shell will therefore have different aspect ratios and the shell thickness will be nonuniform. The Lagrangian for the nanorice particles is diagonal in multipolar indices $l m$. For this reason, the nanorice plasmons can be expressed as bonding and antibonding modes resulting from an interaction between a solid particle plasmon and a cavity plasmon of a common multipolar index $\mathrm{lm}$.

A straightforward application of the plasmon hybridization method to a solid prolate spheroid of aspect ratio coth $\alpha$ gives the following plasmon modes:

$$
\omega_{S, l m}^{2}(\alpha)=\omega_{B}^{2} \frac{P_{l m}^{\prime}(\cosh \alpha) Q_{l m}(\cosh \alpha)}{\varepsilon_{S} P_{l m}^{\prime}(\cosh \alpha) Q_{l m}(\cosh \alpha)-\varepsilon_{E} P_{l m}(\cosh \alpha) Q_{l m}^{\prime}(\cosh \alpha)},
$$

where $\varepsilon_{\mathrm{S}}$ is the background polarizability of the metal and $\varepsilon_{\mathrm{E}}$ is the dielectric permittivity of a possible embedding medium. The plasmon energies of a prolate spheroidal cavity filled with a medium with dielectric permittivity $\varepsilon_{\mathrm{C}}$ take the form:

$$
\omega_{C, l m}^{2}(\alpha)=\omega_{B}^{2} \frac{P_{l m}(\cosh \alpha) Q_{l m}^{\prime}(\cosh \alpha)}{\varepsilon_{S} P_{l m}(\cosh \alpha) Q_{l m}^{\prime}(\cosh \alpha)-\varepsilon_{C} P_{l m}^{\prime}(\cosh \alpha) Q_{l m}(\cosh \alpha)} .
$$

The presence of dielectrics plays a large role in the plasmon frequencies of the solid spheroid and cavity. For the nanorice particles, which contain a hematite core with a dielectric constant of approximately $\varepsilon_{C}=9.5$, the cavity mode lies below the spheroid mode for the transverse polarization for all aspect ratios. For the aspect ratio considered in the present paper, $\varepsilon=4.575$, the cavity plasmon lies above the solid spheroid mode for longitudinal polarization.

The nature of the plasmon modes in a shell particle depends on the relative position of the primitive plasmons. For transverse polarization, the bonding nanorice plasmon is primarily composed of the cavity plasmon while the antibonding mode is solid particle-like. In a shell geometry, the multipolar moments of the cavity plasmons are smaller than those of the solid particle plasmons. In the electrostatic limit, the probability for excitation of a plasmon mode is proportional to the square of its dipole moment. ${ }^{7}$ For this reason, for longitudinal polarization, the bonding plasmon is much brighter than the antibonding plasmon. For the transverse modes, the situation is reversed and the antibonding plasmon is much brighter than the bonding plasmon. 


\section{Reference:}

(1) Ozaki, M.; Kratohvil, S.; Matijevic, E. J. Colloid Interface Sci. 1984, 102, 146-151.

(2) Duff, D. G.; Baiker, A.; Edwards, P. P. Langmuir 1993, 9, 2301-2309.

(3) Westcott, S. L.; Oldenburg, S. J.; Lee, T. R.; Halas, N. J. Langmuir 1998, 14, 5396-5401.

(4) Oldenburg, S. J.; Averitt, R. D.; Westcott, S. L.; Halas, N. J. Chem Phys Lett 1998, 288, 243-247.

(5) Malynych, S.; Luzinov, I.; Chumanov, G. J Phys Chem B 2002, 106, 1280-1285.

(6) Tam, F.; Moran, C. E.; Halas, N. J. J. Phys. Chem. B. 2004, 108, 17290-17294.

(7) Prodan, E.; Nordlander, P. J. Chem. Phys. 2004, 120, 5444-5454.

(8) Vinogradov, S. S.; Smith, P. D.; Vinogradova, E. D. Canonical problems in scattering and potential theory; Chapman \& Hall/CRC: Boca Raton, Florida, 2001. 